Posten operierenden Türken - und vor allem die Auseinandersetzung London - Konstantinopel über die Ausschaltung von Störaktionen der Türken. Diesen, die ihren grundsätzlichen Anspruch auf ganz Südarabien niemals formell aufgaben (S. 16), die Landverbindung zu diesem Teil Arabiens abzuschneiden, war das Hauptziel der Grenzfestlegung der Jahre 1903-1905 (S. 6). Nicht nur in diesem Zusammenhang ist die Feststellung des Verfassers von Bedeutung, daß „der Wille zur Einschränkung imperialer Verpflichtungen die britische Politik spätestens seit Ausbruch des $\mathrm{Bu}-$ renkrieges auch in Detailfragen beherrschte" (S. 21), wenn dem auch die sehr drastische Warnung von Lord Lansdowne vom 5. Mai 1903 hinsichtlich des Persischen Golfs widerspricht Gehrke, der in seinem Überblick über die britisch-jemenitischen Beziehungen auf die beiderseitigen Rechtsansprüche eingeht und auch den Zeitraum 19071920 (u. a. Anglo-Türkische Konvention vom 9. März 1914, S. 95) behandelt hat, insbesondere in den Partien über die letzten Jahre, von einer anderen weitläufigeren Quellenlage auszugehen; er stützt sich vorwiegend auf die in der Dokumentationsstelle für den Modernen Orient zusammengetragenen Materialien und bis 1960 auf die Archivmaterialien des Instituts für Auswärtige Politik in Hamburg. Auf dieser Grundlage gelingt es Gehrke, das verwickelte Spiel in Südarabien deutlich zu machen: Die britischen Versuche, durch eine Föderationsregierung der Scheichs einen ruhigen Übergang der Macht aus britischen in arabische Hände zu erreichen; ihr Scheitern gegenüber den sich an Radikalität überbietenden Nationalisten-Gruppen, vor allem der Flosy und der NLF, die schließlich unter Verdrängung ihrer $\mathrm{Ri}$ valen die Macht an sich riß.

Die doppelte Aktualität des Gehrkeschen Beitrages liegt darin, daß sie nicht nur die Voraussetzungen für die künftigen Gestaltungen in Südarabien aufzeigt; sondern auch die Gefahren erkennen läßt, die den Gebieten am Persischen Golf mit dem Abzug der briti- schen Präsenz bis Ende 1971 drohen: Hier wie dort stehen traditionellen Autoritäten progressive Kräfte gegenüber; hier wie dort herrschen in beiden Lagern Rivalitäten; hier wie dort hängt die Entscheidung weitgehend von energischen Führerpersönlichkeiten $a b$; hier wie dort scheint letzten Endes nicht der vielfach ideell und materiell ferngesteuerte progressive Überschwang, sondern die Solidität der materiellen aber auch der islamischen $\mathrm{Ba}$ sis das Geschehen zu bestimmen.

Conrad Oehlrich

\section{Boutros-Ghali, B.}

L'Organisation de l'Unité Africaine

Librairie Armand Colin, Paris 1969, 197 S.

(Institutions internationales)

Das Verhältnis der Vereinigten Arabischen Republik zur Organisation für Afrikanische Einheit (OAU) war von Anfang an zwiespältig. Einerseits suchte die VAR Einfluß auf die afrikanische Politik und mußte daher die OAU wesentlich unterstützen, andererseits stehen bis heute eine Reihe afrikanischer Staaten, vor allem der frankophonen Gruppe, nicht hinter der von der VAR geförderten Israel-Politik der arabischen Staaten. Die von Nasser schon vor Jahren aufgestellte Formel der drei sich überschneidenden Kreise ägyptischer Außenpolitik: VAR, Arabische Einheit und Afrikanische Einheit, schafft keine Harmonie, sondern Spannungen zwischen den beiden primären Kreisen und dem sekundären Kreis, die in der Praxis zu einem Rückzug der VAR aus der afrikanischen Politik geführt haben. Wenn der Direktor der politikwissenschaftlichen Abteilung der Universität Kairo über die Organisation für Afrikanische Einheit schreibt, darf man daher auf einige Überraschungen gefaßt sein. Das Buch Boutros-Ghalis über die OAU soll eine Studienhilfe sein, die streng unter pädagogischen Gesichtspunkten geschrieben ist und sich auf das Wesentliche beschränkt. So kündigt es der Verfasser jedenfalls im Vorwort an. Diese 
Ankündigung erhöht noch die Erwartung auf das ägyptische Konzentrat der OAU.

Am Anfang des Buches stehen Fragen. Es wird gefragt, ob die OAU eine originelle Konstruktion für eine Regionalorganisation sei, ob die afrikanischen Eliten überhaupt Kraft für originelle Beiträge zum modernen Völkerrecht hätten, ob sie nicht von den innenpolitischen Problemen ihrer Staaten voll absorbiert würden, ob ein neues afrikanisches Völkerrecht entstehe usw. Wenn man die "Contribution à une théorie générale des alliances" von BioutrosGhali kennt, verspricht man sich interessante Antworten auf diese Fragen. Leider werden alle diese Erwartungen enttäuscht. Ein guter Name wird zum Vehikel ägyptischer Propaganda. Die Fragen werden nicht beantwortet.

Aufschlußreich ist schon die Vorgeschichte der OAU, die einleitend skizziert wird. Als wichtigstes Ereignis erscheint hier die Gründung der Casablanca-Gruppe. Die Brazzaville-Gruppe wird mit keinem Wort erwähnt, dafür allerdings die papierene Ghana-GuineaUnion. Die Monrovia-Konferenz ist nicht zu übergehen, ein Wort über die retardierende Rolle der VAR und die dadurch ausgelöste Teilung der CasablancaGruppe in schwarzafrikanische und arabische Staaten sucht man vergeblich.

Nach dieser "wesentlichen" Vorgeschichte findet sich im ersten Teil des Buches „die Ideologie der OAU“. Der Verfasser destilliert sie aus Reden afrikanischer Politiker und verfolgt dabei das Ziel, die elementaren Sätze eines „afrikanischen Völkerrechts“ zu gewinnen. $\mathrm{Da}$ es in Afrika heute an Juristen - und nicht nur an diesen - mangelt, steht man bei der Frage nach afrikanischen Völkerrechtsauffassungen immer wieder vor der Notwendigkeit, Reden von Politikern auf ihren juristischen Gehalt abklopfen zu müssen. Ali A. Mazrui hat auf diese Weise in "Towards a Pax Africana" den Versuch gemacht, eine afrikanische Völkerrechtsdogmatik darzustellen. Dieses Verfahren bleibt ein Notbehelf. Sein Ergebnis widerspricht nur zu oft der Rechtspraxis der afrika- nischen Staaten, und es bleibt dann die stete Frage, ob die Rechtspraxis nur gegenwärtigen $\mathrm{Z}$ wangslagen folgt und die Politiker die wahren Intentionen offenbaren oder ob die Politiker „auf dem Markt" Propaganda getrieben haben und ihren Worten keine rechtliche $\mathrm{Re}$ levanz beizumessen ist. Diese Frage wird noch lange nicht $\mathrm{zu}$ entscheiden sein. Man muß sich ihrer jedoch bewußt bleiben, wenn man die von Boutros-Ghali gewonnenen Erkenntnisse richtig einschätzen will. Der Verfasser weist seine ja wohl vorwiegend studentischen Leser weder auf die Problematik der $\mathrm{Me}$ thode noch auf die Notwendigkeit der Skepsis hin. Ideologische Grundlage des afrikanischen Völkerrechtskreises ist für den Verfasser die „Afrikanische Persönlichkeit". Außerungen zur "Negritude“ werden diesem Topos bedenkenlos untergeordnet. Soweit ist nicht einmal Alex Quaison-Sackey, der Außenminister Nkrumahs, in seinem „Africa Unbound" gegangen. Frankophone Gedanken und Ziele finden in dem Buch so oder so keine Berücksichtigung, es sei denn, daß sie als Belegstücke für die kontinentale Verbreitung ägyptischer Vorstellungen dienen können. So wird die tatsächlich höchst bedeutsame OCAM nur in einem Nebensatz erwähnt.

Die Grundregeln des afrikanischen Völkerrechts sind auf dieser Basis sehr einfach: Volle Gleichheit der afrikanischen Staaten, Nicht-Subversion, Anerkennung der bestehenden afrikanischen Grenzen, afrikanische Streitschlichtung, regionaler Pluralismus, Antikolonialismus, AntiApartheid, Anti-Rassismus, Anti-Neokolonialismus, Bündnisfreiheit, bedingungslose Wirtschaftshilfe. Die Definition des Neokolonialismus ist sehr beachtenswert: Wirtschaftsherrschaft, Kulturherrschaft, Zionismus, Mikronationalismus. Alles dies hat die CasablancaGruppe schon vor Jahren den frankophonen Staaten in der BrazzavilleGruppe vorgeworfen, und noch heute setzen sich diese Staaten gegen solche Vorwände zur Subversion zur Wehr. Auf der fünften Gipfelkonferenz der OAU im Jahre 1967 in Kinshasa hat es 
darüber bedeutsame Erklärungen gegeben. Das wird dem Leser allerdings verschwiegeen.

Der zweite Teil des Buches behandelt die Organisation der OAU in einer knappen Skizze. Der dritte Teil ist ein umfangreicher Dokumentenanhang nebst einer Chronologie der Tagungen von OAU-Gremien. Hierin und in den zahlreichen Zitaten von manchmal schwer zugänglichen Reden, aus denen der erste Teil des Buches kompiliert ist, könnte vielleicht ein Wert der Veröffentlichung als Dokumentation gesehen werden.

Dieter Schröder

\section{Louis Henkin}

How Nations Behave. Law and Foreign Policy. Publ. for the Council on Foreign Relations by Pall Mall Press London 1968 XII, 324 S., 63 sh.

Henkin vertritt in diesem Buch mit Nachdruck die These, daß das internationale (vor allem Völker-)Recht ein relevanter Faktor $\mathrm{zwischenstaatlicher}$ Beziehungen ist, und zwar nicht im Sinne der wohlmeinenden aber wirkungslosen pamphletistischen Forderungen nach "mehr Recht" (nur gelegentlich klingt dieser Ton durch). Seine These ist vielmehr als tatsächliche Feststellung gemeint. Sie ist konkreter, nüchterner und reicht zugleich viel weiter: das Völkerrecht is t ein realer, wirksamer Faktor, der das Verhalten der Staaten ganz wesentlich beeinflußt ("how nations behave"). H. spricht mit der Kompetenz dessen, der lange Jahre hindurch von einer praktischen Tätigkeit im Department of State aus das Staatenverhalten aus großer Nähe und Beteiligung beobachten und dann als akademischer Lehrer (Nachfolger von $\mathrm{Ph}$. C. Jessup an der Columbia Universität) darüber reflektieren konnte. Ausgehend von der - postulierten oder beobachteten Progression in den zwischenstaatlichen Beziehungen von Gewalt über Diplomatie zum Recht stellt er fest, daß dessen tatsächliche Tragweite zwar weit entfernt ist vom Ideal legalistischer Weltverbesserer, aber auch vom Zynismus der „Realisten“, deren Skeptizismus jede Relevanz des Völkerrechts verneint. Bestimmt diese einseitig negative Beurteilung den Staatsmann oder Diplomaten (etwa: es sei unrealistisch und illusionär, Institutionen oder Normen zu schaffen, die ja doch von Dritten mißachtet werden), so ergibt sich leicht eine „Eskalation des Völkerrechtsskeptizismus", die auch das bestehende Rechtsgewebe zerstört. $H$. setzt sich im letzten Kapitel mit den prominentesten „realistischen“ Kritikern des "legalistisch-moralistischen" Momentes in der amerikanischen außenpolitischen Praxis, Kennan und Morgenthau, auseinander. Die mangelnde Schärfe der Grundbegriffe der Realisten wie des "national interest" ermöglicht es, das Völkerrechtsinteresse in eben diese Begriffe einzubeziehen. Es ist ein bloßer Truismus zu sagen, daß Staaten (oder Individuen) die Vor- und Nachteile der Rechtsbefolgung abwägen, sich für die Rechtsverletzung entscheiden und die Konsequenzen in Kauf nehmen mögen. Aber die Realisten gehen davon aus, daß Rechtsbefolgung und Nationalinteresse unüberbrückbare Gegensätze sind, die fast nie zusammenfallen. Sie sehen einen Gewinn für das nationale Interesse praktisch nur in der Rechtsverletzung. Dagegen kann dieser Gewinn sehr gut - wichtig ist die langfristige Perspektive - gerade in der Achtung für das Recht liegen, in der dadurch geförderten wechselseitigen Voraussehbarkeit des Verhaltens und damit der Stabilität der internationalen Beziehungen. Entscheidend ist (263), daß sich selten die eindeutige Alternative: nationales Interesse o d e $\mathrm{r}$ Rechtsbeachtung, stellt, sondern daß der Staatsmann in der Regel $\mathrm{zw}$ wischen konkurrierenden nationalen Interessen wählen muß, zu denen auch die Rechtsbefolgung gehört, selbst wenn die Preisgabe etwaiger unmittelbarer aus einer Verletzung sich ergebender Vorteile "schmerzt“. Das zu übersehen ist die Selbsttäuschung und schädliche Auswirkung der „realistischen" Theorie, die nach $\mathrm{H}$. nicht einmal der Wirklichkeit gerecht wird. Denn Staaten beachten, im obigen Sinne „even if it hurts", das Völkerrecht in wesent- 